\title{
ORIGINAL
}

\section{A Novel Method of DAPI Staining for Differential Diagnosis of Renal Amyloidosis}

\author{
Motokazu Matsuura ${ }^{1,2^{*}}$, Hideharu Abe ${ }^{1}$, Tatsuya Tominaga ${ }^{1}$, Akiko Sakurai', Taichi Murakami', Seiji Kishi', \\ Yoshimi Bando ${ }^{3}$, Jun Minakuchi ${ }^{4}$, Kojiro Nagai ${ }^{1}$, and Toshio Doi ${ }^{1,2}$ \\ ${ }^{1}$ Division of Clinical Laboratory, Tokushima University Hospital, ${ }^{2}$ Department of Nephrology, Institute of Biomedical Sciences, Tokushima \\ University Graduate School, ${ }^{3}$ Division of Pathology, Tokushima University Hospital, ${ }^{4}$ Department of Nephrology, Kawashima Hospital, \\ Tokushima, Japan
}

\begin{abstract}
Amyloidosis is often overlooked because its clinical manifestations can mimic those of more-common diseases. It is important to get a precise diagnosis as early as possible for the prevention of further organ damages. Amyloidosis is a disorder caused by deposition of insoluble abnormal amyloid. The kidney is a frequent site of amyloid deposition. The amyloid fibrils have a characteristic appearance and generate birefringence under polarized light when stained with the Congo red dye. Classification of amyloidosis is based on the precursor protein that forms the amyloid fibrils and the distribution of amyloid deposits as either systemic or localized. Involvement of amyloid fibrils in kidneys mainly occurs as amyloid light-chain (AL) or amyloid A (AA) amyloidosis. The potassium permanganate method with Congo red staining was once used widely to discriminate $\mathrm{AL}$ and $\mathrm{AA}$ amyloidoses, but this method has a problem of false positive results. We found that extracellular and cytoplasmic glomerular 4',6-diamidino-2-phenylindole (DAPI) - positive areas were clearly consistent with amyloid deposition in AL amyloidosis. In contrast, the overlapping staining was not seen in AA amyloidosis. Therefore, we propose that DAPI staining readily distinguishes AL renal amyloidosis from AA renal amyloidosis as a simple and reproducible histochemical method. J. Med. Invest. 64 : 217-221, August, 2017
\end{abstract}

Keywords : amyloidosis, DAPI

\section{INTRODUCTION}

Each year, an estimated 50,000 people worldwide will become afflicted with amyloidosis, with more than 3,000 people receiving the diagnosis in North America alone. Its symptoms are vague and nonspecific, often mimicking those of other common conditions. Amyloidoses are a group of disorders in which soluble proteins aggregate and get deposited extracellularly in tissues as insoluble fibrils, causing progressive organ dysfunction $(1,2)$. More than 20 proteins are known to form amyloid fibrils $(3,4)$. The two most common causes of systemic amyloid deposition are monoclonal immunoglobulin light chains (ALs) and the acute-phase reactant serum amyloid A (AA). AL amyloidosis is associated with plasma cell dyscrasias, including monoclonal gammopathy of undetermined significance, and multiple myeloma. AA amyloidosis is typically secondary to chronic inflammatory conditions, such as rheumatoid arthritis and several other inflammatory diseases. Kidneys are some of the most frequent sites of amyloid deposition in AL, AA, and several hereditary amyloidoses (5).

The diagnosis of renal amyloidosis on kidney biopsies relies on Congo red staining, leading to typical apple-green birefringence under polarized light (5). Once, the potassium permanganate method with Congo red staining, as described by Wright et al. in 1977 (6), had been widely used to discriminate AL and AA amyloidoses. Unfortunately, this method has largely lost the credibility because of false positive results (7). It was recently recommended to use immunohistochemical analysis to detect $\lambda$-light chain, $\kappa$-light

Received for publication February 21, 2017 ; accepted April 20, 2017.

Address correspondence and reprint requests to Motokazu Matsuura, Division of Clinical Laboratory, Tokushima University Hospital, Tokushima, 770-8503, Japan, and Fax : +81-88-633-9245, chain and amyloid A for differential diagnosis of AL and AA amyloidoses (8). Nonetheless, this immunohistochemical examination takes a long time and has a problem with reproducibility due to variations in the protocols and conditions of the antibodies. Therefore, simple and reproducible methods are needed to distinguish AL and AA amyloidoses.

In our previous immunofluorescence staining technique for the detection of a set of immunoglobulins and complements factors for diagnosis of 130 renal biopsy samples at Tokushima University Hospital between 2008 and 2010, we performed staining with $4^{\prime}, 6$ diamidino-2-phenylindole (DAPI) for the detection of nuclei in all samples. In the series of staining of 130 kidney slices, we found that DAPI produces positive staining in the areas of the extracellular matrix and cytoplasm only in tissue slices from patients with renal amyloidosis, and that the DAPI-positive areas reliably coincide with Congo red-positive areas. In the present study, we investigated the utility of staining with DAPI for the diagnosis of renal amyloidosis. This study suggests that a staining method involving the broadly used dye DAPI may serve as a simple and reproducible diagnostic test for renal amyloidosis.

\section{METHODS}

Patients-We investigated 14 cases of glomerular amyloid deposition at Tokushima University Hospital and Kawashima Hospital between 2002 and 2015. Renal amyloidosis were proven by renal biopsy. All of the procedures were performed in accordance with the guidelines of the Helsinki Declaration on Human Experimentation and the Ethical Guidelines on Clinical Research published by the Japanese Health, Labour and Welfare Ministry. This study was approved by the Ethics Committee of Tokushima University and Kawashima Hospital, and informed consent was obtained from all 
patients.

Laboratory examination-Ccreatinine in serum was measured by a enzymatic method. Twenty four-hour urine protein in $\mathrm{g} /$ day was determined to multiply the 24 -hour urine volume by the urine protein concentration measured using a pyrocatechol violet molybdate dye-binding method. Serum amyloid A protein (SAA) was measured by a latex agglutination immunoassay method.

Histology-Histopathological studies were performed on human tissues. Kidney specimens $(n=14)$ were obtained from renal biopsies. Kidney tissue blocks for light microscopy examination were fixed with Dubosq-Brazil's solution and embedded in paraffin. Amyloid staining was performed with Congo-red, exhibited applegreen birefringence on polarizing microscopy, and confirmed the reaction of the potassium permanganate digestion.

Immunohistochemistry-Kidney sections were processed for immunohistochemistry following standard procedures. DubosqBrazil's solution-fixed and paraffin-embedded kidney tissue blocks were used. Before dyeing them, tissue sections $(2 \mu \mathrm{m})$ were rehydrated and treated with HistoVT One (Nacalai Tesque Inc., Kyoto Japan) for $20 \mathrm{~min}$ at $98^{\circ} \mathrm{C}$. To perform DAPI- $\lambda / \kappa$ double staining, sections were stained with FITC-conjugated anti-human lambda light chains antibody (1: 40 dilution) (Dako, Kyoto, Japan) or antihuman kappa light chains antibody (1: 40 dilution) (Dako, Kyoto, Japan) for $120 \mathrm{~min}$ at room temperature and then incubated with DAPI (1 : 1000 dilution) (Wako, Osaka, Japan) for $30 \mathrm{~min}$ at room temperature. To perform DAPI-amyloid A double staining, sections were stained with anti-human amyloid A antibody (1:100 dilution) (Dako, Kyoto, Japan) for $90 \mathrm{~min}$ at room temperature followed by incubation with the appropriate secondary antibodies conjugated with FITC, and then stained with DAPI (1 : 1000 dilution) for $30 \mathrm{~min}$ at room temperature. To perform DAPI-Propidium lodide double staining, sections were stained with DAPI (1: 1000 dilution) overnight at $4^{\circ} \mathrm{C}$ and then stained with Propidium lodide (1: 500 dilution) (KPL, MD, USA) for $5 \mathrm{~min}$ at room temperature.

\section{RESULTS}

In an attempt to clarify the differential diagnosis of amyloidosis, we conducted histochemical evaluation of amyloid deposition in kidneys. The clinical profile of 14 patients is shown in Table 1 . The presence of $\lambda$-light chain monoclonal proteins in serum and/or urine was examined by immunoelectrophoresis tests. As a result, $\lambda$ light chain monoclonal proteins were detected in patients $1-5,7,8$, and 10 . As for patient 6 , these tests were not performed because of sudden death. Monoclonal proteins were not detected by these tests in the patient 9. Patients with rheumatoid arthritis (11 to 14) showed high levels of serum amyloid A. Patients 11 and 14 had advanced renal failure.

The pathological profile of the patients is shown in Table 2. All patients had Congo red-positive staining in glomeruli and showed apple-green birefringence under polarized light. In addition, electron microscopic analysis revealed amyloid fibrils in glomeruli in all patients. According to the potassium permanganate method with Congo red staining, kidney slices from patients 1-9 and 10-14 were potassium permanganate-resistant and potassium permanganate-sensitive, respectively. Patients 11-14 had deposition of amyloid A in glomeruli. In patient 3 , positive staining of $\lambda$-light chain in glomeruli was detected in addition to amyloid A. In patient 10 , all staining yield negative results. By bone marrow biopsy, multiple myeloma was ruled out for patients $1-10$. As for patient 6 , the bone marrow biopsy was not performed due to sudden death. Patient 3 did not have any chronic inflammatory conditions to cause AA amyloidosis. Although patient 10 received a diagnosis of monoclonal gammopathy of undetermined significance, which was close to AL amyloidosis, the deposition of paraproteins in glomeruli could not be detected by immunohistochemical and mass spectrometric analyses.

The results of DAPI staining in these patients are shown in Table 3. Glomerular DAPI-positive areas as accompanied with nuclei were observed in AL amyloidosis, indicating that DAPIpositive staining areas except for nuclei are consistent with amyloid deposition areas (Fig. 1g, h). In contrast, the consistency of overlapping staining patterns was not seen in AA amyloidosis (Fig. 1c, f, i). The overlapping staining patterns with amyloid deposition were not observed with the staining by means of another nuclear staining

Table 1. The clinical profiles of 14 patients

\begin{tabular}{|c|c|c|c|c|c|c|c|}
\hline \multirow[t]{2}{*}{ Patient } & \multirow{2}{*}{$\begin{array}{l}\text { Age(years) } \\
\text { Sex }\end{array}$} & \multirow{2}{*}{$\begin{array}{l}\text { Proteinuria } \\
\text { (g/day) }\end{array}$} & \multirow{2}{*}{$\underset{(\mathrm{mg} / \mathrm{dl})}{\mathrm{Cr}}$} & \multicolumn{2}{|c|}{ Immunoelectrophoresis } & \multirow{2}{*}{$\begin{array}{c}\text { SAA } \\
(\mu \mathrm{g} / \mathrm{ml})\end{array}$} & \multirow{2}{*}{$\begin{array}{c}\text { Underlying } \\
\text { disease }\end{array}$} \\
\hline & & & & (Blood) & (Urine) & & \\
\hline 1 & $69 \mathrm{M}$ & 7.3 & 1.01 & neg & BJP- $\lambda$ & $\mathrm{N} / \mathrm{P}$ & \\
\hline 2 & $76 \mathrm{M}$ & 4.8 & 1.61 & $\operatorname{IgG}-\lambda$ & $\operatorname{IgG}-\lambda$ & $\mathrm{N} / \mathrm{P}$ & \\
\hline 3 & $39 \mathrm{~F}$ & 2.5 & 0.61 & neg & BJP- $\lambda$ & $\mathrm{N} / \mathrm{P}$ & \\
\hline 4 & $55 \mathrm{M}$ & 3.9 & 1.68 & $\operatorname{Ig} \mathrm{A}-\lambda$ & $\operatorname{IgA}-\lambda$ & $\mathrm{N} / \mathrm{P}$ & \\
\hline 5 & $83 \mathrm{M}$ & 5.2 & 0.83 & $\operatorname{IgG}-\lambda$ & IgG- $\lambda$ & $\mathrm{N} / \mathrm{P}$ & \\
\hline 6 & $77 \mathrm{~F}$ & 3.1 & 0.70 & $\mathrm{~N} / \mathrm{P}$ & $\mathrm{N} / \mathrm{P}$ & $\mathrm{N} / \mathrm{P}$ & \\
\hline 7 & $81 \mathrm{~F}$ & 2.2 & 1.05 & $\operatorname{IgG}-\lambda$ & BJP- $\lambda$ & $\mathrm{N} / \mathrm{P}$ & \\
\hline 8 & $83 \mathrm{M}$ & 4.2 & 0.98 & neg & BJP- $\lambda$ & $\mathrm{N} / \mathrm{P}$ & \\
\hline 9 & $62 \mathrm{M}$ & 2.9 & 1.05 & neg & neg & $\mathrm{N} / \mathrm{P}$ & \\
\hline 10 & $67 \mathrm{~F}$ & 1.1 & 0.76 & $\operatorname{IgG}-\lambda$ & neg & $\mathrm{N} / \mathrm{P}$ & \\
\hline 11 & $50 \mathrm{~F}$ & 3.7 & 4.37 & $\mathrm{~N} / \mathrm{P}$ & $\mathrm{N} / \mathrm{P}$ & 276 & $\mathrm{RA}$ \\
\hline 12 & $69 \mathrm{~F}$ & 1.9 & 1.59 & neg & neg & 61.7 & $\mathrm{RA}$ \\
\hline 13 & $67 \mathrm{~F}$ & 6.0 & 0.64 & $\mathrm{~N} / \mathrm{P}$ & $\mathrm{N} / \mathrm{P}$ & 714.9 & $\mathrm{RA}$ \\
\hline 14 & $34 \mathrm{~F}$ & 6.5 & 2.34 & $\mathrm{~N} / \mathrm{P}$ & neg & 882 & $\mathrm{RA}$ \\
\hline
\end{tabular}

$\mathrm{M}$ : male ; $\mathrm{F}$ : female $; \mathrm{Cr}$ : serum creatinine ; neg : negative result

$\mathrm{N} / \mathrm{P}$ : notperformed ; SAA : serum amyloid A protein ; RA : rheumatoid arthritis 
Table 2. The pathological profiles of 14 patients

\begin{tabular}{|c|c|c|c|c|c|}
\hline Patient & Congo-red & $\begin{array}{c}\text { Potassium } \\
\text { permanganate } \\
\text { method }\end{array}$ & $\begin{array}{c}\text { Glomerular } \\
\lambda / \kappa \\
\text { lightchain }\end{array}$ & $\begin{array}{c}\text { Glomerular } \\
\text { amyloidA }\end{array}$ & $\begin{array}{c}\text { Glomerular } \\
\text { amyloid fibrils } \\
\text { (Electron microscopy) }\end{array}$ \\
\hline 1 & pos & $\mathrm{r}$ & $\mathrm{pos} / \mathrm{neg}$ & $\mathrm{neg}$ & + \\
\hline 2 & $\mathrm{pos}$ & $\mathrm{r}$ & $\mathrm{pos} / \mathrm{neg}$ & $\mathrm{neg}$ & $\mathrm{N} / \mathrm{P}$ \\
\hline 3 & $\mathrm{pos}$ & $\mathrm{r}$ & $\mathrm{pos} / \mathrm{neg}$ & $\mathrm{pos}$ & $\mathrm{N} / \mathrm{P}$ \\
\hline 4 & $\mathrm{pos}$ & $\mathrm{r}$ & $\mathrm{pos} / \mathrm{neg}$ & $\mathrm{neg}$ & + \\
\hline 5 & $\mathrm{pos}$ & $\mathrm{r}$ & $\mathrm{pos} / \mathrm{neg}$ & $\mathrm{neg}$ & + \\
\hline 6 & $\mathrm{pos}$ & $\mathrm{r}$ & $\mathrm{pos} / \mathrm{neg}$ & $\mathrm{neg}$ & + \\
\hline 7 & $\mathrm{pos}$ & $\mathrm{r}$ & $\mathrm{pos} / \mathrm{neg}$ & $\mathrm{neg}$ & + \\
\hline 8 & $\mathrm{pos}$ & $\mathrm{r}$ & $\mathrm{pos} / \mathrm{neg}$ & $\mathrm{neg}$ & + \\
\hline 9 & $\mathrm{pos}$ & $\mathrm{r}$ & $\mathrm{pos} / \mathrm{neg}$ & $\mathrm{neg}$ & + \\
\hline 10 & $\mathrm{pos}$ & $\mathrm{s}$ & $\mathrm{neg} / \mathrm{neg}$ & $\mathrm{neg}$ & + \\
\hline 11 & $\mathrm{pos}$ & $\mathrm{s}$ & $\mathrm{neg} / \mathrm{neg}$ & $\mathrm{pos}$ & + \\
\hline 12 & $\mathrm{pos}$ & $\mathrm{s}$ & $\mathrm{neg} / \mathrm{neg}$ & $\mathrm{pos}$ & $\mathrm{N} / \mathrm{P}$ \\
\hline 13 & $\mathrm{pos}$ & $\mathrm{s}$ & $\mathrm{neg} / \mathrm{neg}$ & $\mathrm{pos}$ & $\mathrm{N} / \mathrm{P}$ \\
\hline 14 & $\mathrm{pos}$ & $\mathrm{s}$ & $\mathrm{neg} / \mathrm{neg}$ & $\mathrm{pos}$ & \\
\hline
\end{tabular}

pos : positive result ; neg : negative result ; $\mathrm{s}$ : sensitive ; $\mathrm{r}$ : resistant

+ : present ; - : absent; N/P : not performed
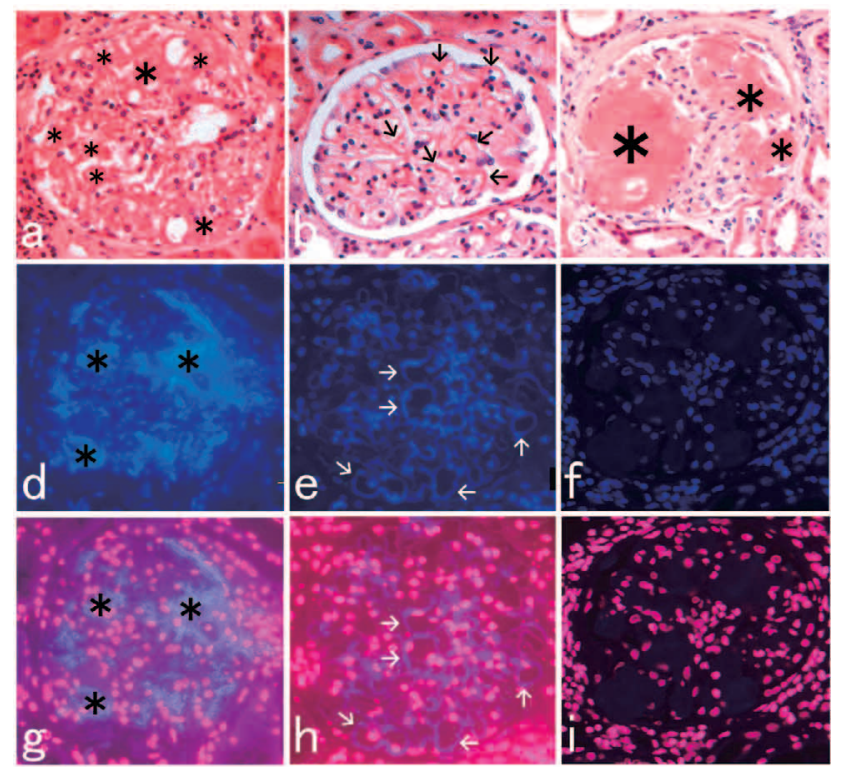

Figure 1 Microscopic appearance of the glomeruli with deposition of amyloid. (a-c) Congo-red staining. (d-f) DAPI staining. (g-i) Double staining of DAPI (blue) and Propidium Iodide (PI) (red). In the glomeruli, amyloid deposits are present in the mesangial matrix (asterisks) and along the glomerular basement membrane (arrows). (a, d, g) Patient 1 ; AL amyloidosis with deposition in mesangiocapillary pattern. (b, e, h) Patient 7 ; AL amyloidosis with deposition in perimembranous pattern. (c, f, i) Patient 11; AA amyloidosis. Original magnification for all panels was $\times 400$.

reagent propidium iodide $(\mathrm{PI})$.

Thus, DAPI-PI double staining was performed on 13 patients ; DAPI and $\lambda$ - or $\kappa$-light chain double staining was conducted on five patients with AL amyloidosis ; and DAPI-amyloid A double staining, on two patients with AA amyloidosis. Consequently, DAPI-
Table 3.

\begin{tabular}{|c|c|c|}
\hline Patient & Diagnosis & $\begin{array}{c}\text { DAPI-positive areas } \\
\text { without nuclei }\end{array}$ \\
\hline 1 & $\mathrm{AL}$ & + \\
\hline 2 & $\mathrm{AL}$ & + \\
\hline 3 & $\mathrm{AL}$ & + \\
\hline 4 & $\mathrm{AL}$ & + \\
\hline 5 & $\mathrm{AL}$ & + \\
\hline 6 & $\mathrm{AL}$ & + \\
\hline 7 & $\mathrm{AL}$ & + \\
\hline 8 & $\mathrm{AL}$ & + \\
\hline 9 & $\mathrm{AL}$ & + \\
\hline 10 & $\mathrm{MGUS}$ & partially present \\
\hline 11 & $\mathrm{AA}$ & - \\
\hline 12 & $\mathrm{AA}$ & - \\
\hline 13 & $\mathrm{AA}$ & - \\
\hline 14 & $\mathrm{AA}$ & - \\
\hline
\end{tabular}

AL : AL amyloidosis ; AA : AA amyloidosis

MGUS : monoclonal gammopathy of undetermined significance + : present ; - : absent

positive areas without nuclei corresponded to the deposition of paraprotein, which was the $\lambda$-light chain monoclonal protein in every patient with AL amyloidosis (Fig. 2a-c).

\section{DISCUSSION}

Amyloidosis is defined as pathological accumulation of extracellular proteins that adopt a $\beta$-pleated configuration and share histochemical characteristics and fibrillary ultrastructure. These proteins are resistant to degradation. These substances share morphological, ultrastructural, and staining features, but they have different chemical structure. The diagnosis of amyloidosis requires histological demonstration of amyloid deposits. Many pathologists 

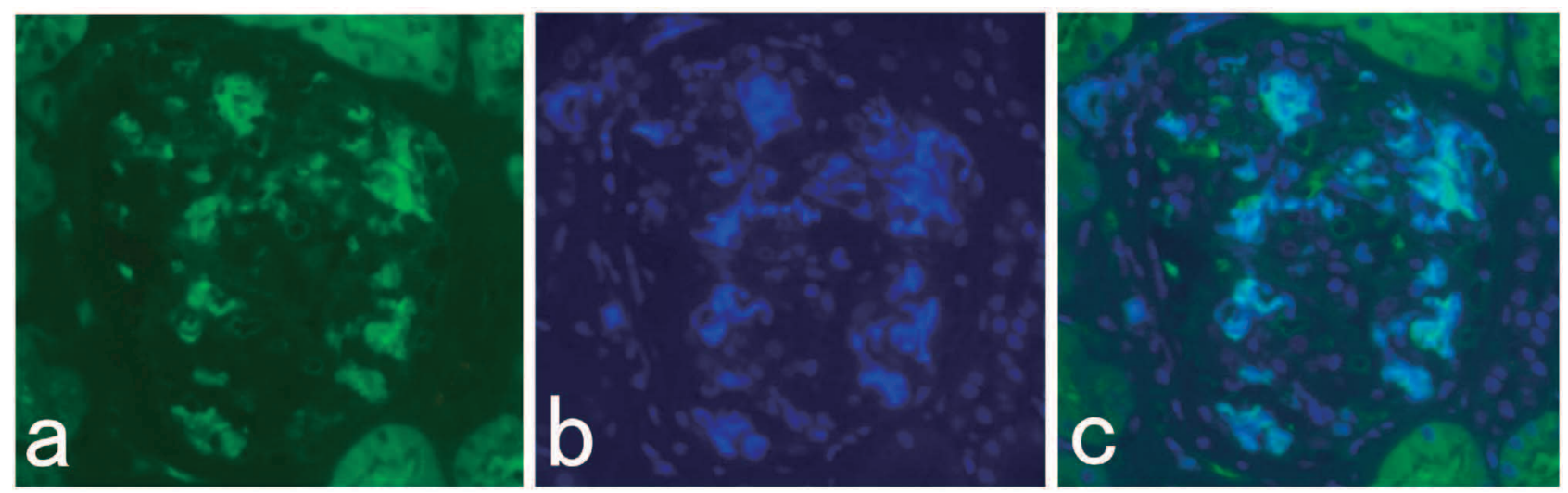

Figure 2 Cytoplasmic staining with DAPI is coincided with the deposition of $\lambda$ light chain. (a) Immunofluorescence stainings for $\lambda$ light chain. (b) DAPI staining. (c) Overlay images of DAPI (blue) and $\lambda$ light chain (green). (a-c) Patient 1 ; AL amyloidosis with deposition in mesangiocapillary pattern. Original magnification for all panels was $\times 400$.

use Congo red to make a diagnosis of amyloidosis and state the common opinion that Congo red-stained amyloid in polarized light shows apple-green birefringence, sometimes called apple-green dichroism.

Patients with primary amyloidosis can be classified into two groups : one with potassium permanganate-sensitive and one with potassium permanganate-resistant amyloid deposits. These two groups correlate with the clinical classification of typical organ distribution (presenting with nephropathy) and atypical organ distribution (presenting with cardiomyopathy, nephropathy, and glossopathy) and the expected presence of amyloid AA or amyloid AL. This histochemical method for differentiating amyloid AA from AL was introduced by Wright et al. (6). The method is based on the affinity of amyloid for Congo red dye after exposure to potassium permanganate and dilute sulfuric acid. Because potassium permanganate Congo red staining has been barely used after 2001 (7), it was recently recommended to use immunohistochemical analysis to detect $\lambda$-light chain, $\kappa$-light chain, and amyloid $A$ as a diagnostic tool for the discrimination of AL and AA amyloidoses (8). On the other hand, this immunohistochemical examination takes a long time and has a problem with reproducibility due to variations in the protocols and conditions of antibodies. Therefore, simple and reproducible methods are needed to distinguish $\mathrm{AL}$ and $\mathrm{AA}$ amyloidoses.

Our study revealed that extracellular and cytoplasmic glomerular DAPI-positive areas without nuclei are clearly consistent with amyloid deposition in $\mathrm{AL}$ amyloidosis. In contrast, the overlapping staining was not seen in AA amyloidosis. These results suggest that DAPI-staining can be a useful way to discriminate AL and AA amyloidoses. It is also well known that glomerular amyloid deposition is categolized into several types : 1) a mesangiocapillary type with diffuse amyloid deposition in the mesangium and along both sides of the glomerular basement membrane ; 2) a perimembranous type principally involving the subepithelial side of the basement membrane ; 3$)$ a mesangial nodular type showing nodular mesangial deposits ; 4) and a hilar type showing amyloid deposits in hilar arterioles (9-11). We demonstrated here that DAPI-PI double staining clarifies the difference in amyloid deposition types more effectively. Moreover, considering the result of patient 10 , DAPI might detect tiny amount of amyloid deposition that is resistant to the potassium permanganate method, the immunohistochemical method, and the mass spectrometry. DAPI (4',6diamidino-2-phenylindole) is a blue fluorescent nuclear stain broadly used in fluorescence microscopy assays (12). It is known that DAPI binds to some kind of protein (13-15). Recently, many molecular probes for amyloid fibrils have been reported $(16,17)$. Some $2-$ phenylindole derivatives are known as such probes (18), but as far as we know, there are no reports that DAPI can be used as a diagnostic dye for renal amyloidosis. Although the molecular binding mechanism is unknown in detail, the following is one possibility. It is known that AA-amyloid protein has a higher proportion of arginine than AL-amyloid protein does $(6,19,20)$. Because DAPI and arginine both have basic $\mathrm{pKa}$, they carry a positive charge and repel each other under physiological conditions. This may be one of the reasons why DAPI does not bind to AA amyloid protein.

In conclusion, we demonstrated here that widely used DAPI staining is more rapid and easier to perform than the potassium permanganate method with Congo red staining and the immunohistochemistry to detect amyloid paraprotein. DAPI can detect even a small deposit of amyloid paraprotein for testing of resistance to the potassium permanganate method and to immunohistochemical analysis. Moreover, DAPI staining revealed these deposits more clearly than Congo red staining did in most patients in this study. These results suggested that the staining method with popular dye DAPI may be rapid and easy way to discriminate AL and AA amyloidoses. It is imperative for clinicians and pathologists to consider amyloidosis as part of their differential diagnosis. Given the simple and reproducible staining method for amyloid proteins, testing for the disease becomes easier too. Early accurate diagnosis is necessary for patients with amyloidosis, and they may benefit from the new diagnostic procedure that is available to improve and extend life. We cannot deny that this study only shows a preliminary result because there were only a limited number of such patients available at our institution. Nonetheless, we believe that this result represents an interesting finding, and that confirmation on a large sample size should make this conclusion firmer.

\section{FUNDING}

This study was supported by Grants-in-Aid for Scientific Research (C) (24591201) and the Kidney Foundation of Japan (JKFB16-11).

\section{CONFLICT OF INTEREST}

The authors declare no competing financial interests. 


\section{ACKNOWLEDGMENT}

We thank H. Nakagawa, A. Ochi, S. Hayashi, and T. Sasaki for their excellent technical assistance.

\section{AUTHOR CONTRIBUTIONS}

The author(s) have made the following declarations regarding their contributions : M.M., H.A., T.T. and T.D. designed this study. M.M., H.A., A.S., T.M., S.K., Y.B., J.M. and K.N. acquired the data. M.M., H.A., T.T., A.S., Y.B. and T.D. performed the analysis and interpretation of data. M.M. and H.A. wrote the paper. M.M., H.A. and T.D. supported the project financially.

\section{REFERENCES}

1. Sipe JD, Cohen AS : Review : History of the Amyloid Fibril. J Struct Biol $130: 88-98,2000$

2. Obici L, Raimondi S, Lavatelli F, Bellotti V, Merlini G : Susceptibility to AA amyloidosis in rheumatic diseases : A critical overview. Arthritis Rheum 61 : 1435-1440, 2009

3. Sipe JD, Benson MD, Buxbaum JN, Ikeda S, Merlini G, Saraiva MJM, Westermark P : Amyloid fibril protein nomenclature : 2010 recommendations from the nomenclature committee of the International Society of Amyloidosis. Amyloid 17 : 101104, 2010

4. Pinney JH, Lachmann HJ : Systemic AA amyloidosis. Subcell Biochem 65 : 541-564, 2012

5. Dember LM : Amyloidosis-associated kidney disease. J Am Soc Nephrol $17: 3458$-3471, 2006

6. Wright JR, Calkins E, Humphrey RL : Potassium permanganate reaction in amyloidosis : A histologic method to assist in differentiating forms of this disease. Lab Invest 36 : 274-281, 1977

7. Vowles GH, Francis RJ : Amyloid. In : Bancroft JD, Gamble M, eds. Theory and Practice of Histological Techniques, 5th ed. Churchill Livingstone, London, 2001, pp.303-324

8. Schönland SO, Hegenbart U, Bochtler T, Mangatter A, Hansberg M, Ho AD, Lohse P, Röcken C: Immunohistochemistry in the classification of systemic forms of amyloidosis : A systematic investigation of 117 patients. Blood 119 : 488493, 2012
9. Shiiki H, Shimokama T, Yoshikawa Y, Toyoshima H, Kitamoto $\mathrm{T}$, Watanabe $\mathrm{T}$ : Renal amyloidosis. Correlations between morphology, chemical types of amyloid protein and clinical features. Virchows Arch A Pathol Anat Histopathol 412 : 197204, 1988

10. Sen S, Sarsik B : A proposed histopathologic classification, scoring, and grading System for renal amyloidosis. Standardization of renal amyloid biopsy report. Arch Pathol Lab Med 134 : 532-544, 2010

11. Castano E, Palmer MB, Vigneault C, Luciano R, Wong S, Moeckel $\mathrm{G}$ : Comparison of amyloid deposition in human kidney biopsies as predictor of poor patient outcome. BMC Nephrology $16: 64,2015$

12. Kapuscinski J : DAPI : a DNA-specific fluorescent probe. Biotechnic and Histochemistry $70: 220-233,1995$

13. Bonne D, Heusèle C, Simon C, Pantaloni D : 4', 6-Diamidino-2phenylindole, a fluorescent probe for tubulin and microtubules. J Biol Chem 260 : 2819-2825, 1985

14. Tijssen TPF, Beekes HW, Van Steveninck J : Localization of polyphosphates in Saccharomyces fragilis, as revealed by 4, 6 diamidino-2-phenylindole fluorescence. Biochimica Biophysica Acta $721: 394-398,1982$

15. Katouzian-Safadi M, Cremet J, Charlier M : Limitation of DNA-4',6-diamidine-2-phenylindole assay in the presence of an excess of tRNA. Anal Biochem 176 : 416-419, 1989

16. Peter K, Nilsson R: Small organic probes as amyloid specific ligands-Past and recent Molecular scaffolds. FEBS Letters 583 : 2593-2599, 2009

17. Groenning $\mathrm{M}$ : Binding mode of Thioflavin $\mathrm{T}$ and other molecular probes in the context of amyloid fibrils - current status. J Chem Biol 3 : 1-18, 2010

18. Fu H, Yu L, Cui M, Zhang J, Zhang X, Li Z, Wang X, Jia J, Yang Y, Yu P, Jia H, Liu B : Synthesis and biological evaluation of ${ }^{18} \mathrm{~F}$-labled 2-phenylindole derivatives as PET imaging probes for $\mathrm{b}$-amyloid plaques. Bioorganic \& Medicinal Chemistry $21: 3708-3714,2013$

19. Wright JR, Humphrey RL, Calkins E : Different molecular forms of amyloid histologically distinguished by susceptibility or resistance to trypsin digestion. In : Wegelins O, Pasternack A, eds. Amyloidosis. Academic Press, London, 1976, pp51-59

20. van Rijswijk MD, van Heusden $\mathrm{CW}$ : The Potassium Permanganate Method: Areliable method for differentiating amyloid AA from other forms of amyloid in routine laboratory practice. Am J Pathol 97 : 43-58, 1979 